\title{
THE ASTROPHYSICAL JOURNAL SUPPLEMENT
}

\author{
AUTHOR INDEX
}

VOLUME 90

\section{JANUARY TO FEBRUARY}

ANDERSON, Martha C. Do Relativistic Electrons either Gain or Lose Energy, outside of Extragalactic Nuclei? Lawrence Rudnick, Debora M. Katz-Stone, \& Martha C. Anderson. 90, 955, 15-F6 (1994)

Arons, Jonathan. Relativistic Particle Acceleration in Plerions. Jonathan Arons \& Marco Tavani. 90, 797, 13-F1 (1994)

Aschwanden, Markus J. Puised Acceleration in Solar Flares. Markus J. Aschwanden, Amold O. Benz, Brian R. Dennis, \& MukulR. Kundu. 90, 631, 11-E11 (1994)

Aurass, H. The Flare of 1989 September 9 09:09 UT: Does Coronal Loop Collision Initiate Efficient Gamma-Ray Emission? H. Aurass, A. Hofmann, \& E. Rieger. 90, 707, 12-E6 (1994)

AXFORD, W. I. The Origins of High-Energy Cosmic Rays. W. I. Axford. 90, 937, 15-D11 (1994)

BAKER, DANIEL N. Evidence for Particle Acceleration during Magnetospheric Substorms. Ramon E. Lopez \& Daniel N. Baker. 90, 531, 10-C13 (1994)

BALL, LEwIS. Radio Emission from SNR 1987A. J. G. Kirk, P. Duffy, \& Lewis Ball. 90, 807, 13-F13 (1994)

Electron Acceleration in Short-lived Radio Transients. Lewis Ball. 90, 889, 14-G4 (1994)

BALOGH, A. Drift Acceleration at Interplanetary Shocks. G. Erdös \& A. Balogh. 90, 553, 10-F1 (1994)

Baring, Matthew G. Monte Carlo Simulations of Particle Acceleration at Oblique Shocks. Matthew G. Baring, Donald C. Ellison, \& Frank C. Jones. 90, 547, 10-E6 (1994)

Gamma-Ray Burst Spectral Breaks and Source Beaming. Matthew G. Baring. 90, 899, 15-A3 (1994)

Barnbaum, Cecilia. A High-Resolution Spectral Atlas of Carbon Stars. Cecilia Barnbaum. 90, 317, 5-G10 (1994)

BATCHELOR, DAVID. Another Clue About Particle Acceleration in Impulsive Hard X-Ray/Microwave Bursts. David Batchelor. 90, 697, 12-D7 (1994)

Becker, Peter A. Relativistic Particle Transport in Hot Accretion Disks. Peter A. Becker, Menas Kafatos, \& Michael Maisack. 90, 949, 15-E13 (1994)

Bennett, $K$. Gamma Rays from the Geminga Pulsar: Variations with Time and Phase. I. A. Grenier, K. Bennett, R. Buccheri, M. Gros, R. N. Henriksen, W. Hermsen, G. Kanbach, \& B. Sacco. 90, 813, 13-G6 (1994)

Pulsar Studies with COMPTEL. K. Bennett, M. Busetta, A. Carramiñana, R. Diehl, G. Lichti, V. Schönfelder, A. Strong, W. Hermsen, L. Kuiper, A. Connors, J. Ryan, \& R. Buccheri. 90, 823, 14-A5 (1994)

BENZ, ARNold O. Pulsed Acceleration in Solar Flares. Markus $J$. Aschwanden, Amold O. Benz, Brian R. Dennis, \& Mukul R. Kundu. 90, 631, 11-E11 (1994)

BERG, D. Polarized One-Quantum Annihilation in Strong Magnetic Fields as a Process for Particle Deceleration in Neutron Star Atmospheres. D. Berg, M. Mentzel, \& G. Wunner. 90, 905, 15-A10 (1994)

BiretTA, J. A. Hubble Space Telescope Observations of Synchrotron Jets. W. B. Sparks, J. A. Biretta, \& F. Macchetto. 90, 909, 15-B1 (1994)

BLANDFORD, R. D. Particle Acceleration Mechanisms. $R$. $D$. Blandford. 90, 515, 10-B7 (1994)

Blumenthal, GeORge R. Do Gamma-Ray Bursts Originate from an Extended Galactic Halo of High-Velocity Neutron Stars? Dieter $H$. Hartmann, Lawrence E. Brown, Lih-Sin The, Eric V. Linder, Vahe Petrosian, George R. Blumenthal, \& Kevin C. Hurley. 90, 893, 14-G10 (1994)
Brown, LaWrence E. Do Gamma-Ray Bursts Originate from an Extended Galactic Halo of High-Velocity Neutron Stars? Dieter $H$. Hartmann, Lawrence E. Brown, Lih-Sin The, Eric V. Linder, Vahe Petrosian, George R. Blumenthal, \& Kevin C. Hurley. 90, 893, 14-G10 (1994)

BucCHERI, R. Gamma Rays from the Geminga Pulsar: Variations with Time and Phase. I. A. Grenier, K. Bennett, R. Buccheri, M. Gros, R. N. Henriksen, W. Hermsen, G. Kanbach, \& B. Sacco. 90, 813, 13-G6 (1994)

See BENNETT, K., et al. Pulsar Studies with COMPTEL.

Burke, B. F. The Parkes-MIT-NRAO (PMN) Surveys. III. Source Catalog for the Tropical Survey $\left(-29^{\circ}<\delta<-9^{\circ} .5\right)$. Mark $R$. Griffith, Alan E. Wright, B. F. Burke, \& R. D. Ekers. 90, 179, 3-C6 (1994)

Busetta, M. See Bennett, K., et al. Pulsar Studies with COMPTEL.

Carraminana, A. See Bennett, K., et al. Pulsar Studies with COMPTEL

Cecatro, J. R. Solar Simple Bursts Observed with High Spectral Resolution in the $18-23 \mathrm{GHz}$ Range. H. S. Sawant, $R$. R. Rosa, J. R. Cecatto, \& N. Gopalswamy. 90, 693, 12-D2 (1994)

CHEN, HuA. Stellar Density Enhancements Associated with IRAS Sources in L1641. Hua Chen \& Alan T. Tokunaga. 90, 149, 2-G12 (1994)

CHENG, K. S. Unpulsed High-Energy Radiation from the Crab Pulsar and Nebula. W. M. Cheung \& $K$. S. Cheng. 90, 827, 14-A11 (1994)

Cheung, W. M. Unpulsed High-Energy Radiation from the Crab Pulsar and Nebula. W. M. Cheung \& K. S. Cheng. 90, 827, 14-A11 (1994)

Condon, J. J. The Parkes-MIT-NRAO (PMN) Map Catalog of Radio Sources Covering $-88^{\circ}<\delta<-37^{\circ}$ at $4.85 \mathrm{GHz}$. P. C. Gregory, J. D. Vavasour, W. K. Scott, \& J. J. Condon. 90, 173, 3-B12 (1994)

Connors, A. See BEnNeTt, K., et al. Pulsar Studies with COMPTEL.

CORNIDE, $\mathrm{M}$. Ca II $\mathrm{H}$ and $\mathrm{K}$ and $\mathrm{H} \alpha$ Emissions in Chromospherically Active Binary Systems (RS Canum Venaticorum and BY Draconis). M. J. Fernández-Figueroa, D. Montes, E. De Castro, \& M. Comide. 90, 433, 8-B6 (1994)

Côté, PATrick. A Search for Binaries in the Globular Cluster NGC 3201. Patrick Côté, Douglas L. Welch, Philippe Fischer, G. S. Da Costa, Peter Tamblyn, Patrick Seitzer, \& M. J. Irwin. 90, 83, 2-BS (1994)

DA CosTA, G. S. A Search for Binaries in the Globular Cluster NGC 3201. Patrick Côté, Douglas L. Welch, Philippe Fischer, G. S. Da Costa, Peter Tamblyn, Patrick Seitzer, \& M. J. Irwin. 90, 83, 2-B5 (1994)

D'AnTona, Francesca. New Pre-Main-Sequence Tracks for $M \leq 2.5 M \odot$ as Tests of Opacities and Convection Model. Francesca D'Antona \& Italo Mazzitelli. 90, 467, 8-D14 (1994)

De CASTRo, E. Ca II $\mathrm{H}$ and $\mathrm{K}$ and $\mathrm{H} \alpha$ Emissions in Chromospherically Active Binary Systems (RS Canum Venaticorum and BY Draconis). M. J. Fernández-Figueroa, D. Montes, E. De Castro, \& M. Cornide. 90, 433, 8-B6 (1994)

DE JAGER, O. C. Evidence for Particle Acceleration in a Magnetized White Dwarf from Radio and Gamma-Ray Observations. O. C. De Jager. 90, 775, 13-D3 (1994)

Dennis, Brian R. Pulsed Acceleration in Solar Flares. Markus $J$. Aschwanden, Arnold O. Benz, Brian R. Dennis, \& Mukul R. Kundu. 90, 631, 11-E11 (1994)

Dermer, Charles D. On the Location of the Acceleration and Emission Sites in Gamma-Ray Blazars. Charles D. Dermer \& Reinhard Schlickeiser. 90, 945, 15-E7 (1994) 
Desch, Michael D. Jupiter Radio Bursts and Particle Acceleration. Michael D. Desch. 90, 541, 10-D10 (1994)

DieHL, R. See BenNetT, K., et al. Pulsar Studies with COMPTEL.

DoRfi, E. A. Evolution of Supernova Remnants with Cosmic Rays and Radiative Cooling. E. A. Dorfi. 90, 841, 14-B14 (1994)

Dröge, WolfGang. Transport of Solar Energetic Particles. Wolfgang Dröge. 90, 567, 10-G3 (1994)

DufFy, P. Radio Emission from SNR 1987A. J. G. Kirk, P. Duffy, \& Lewis Ball. 90, 807, 13-F13 (1994)

Duffy, Peter. Bohm Diffusion and Cosmic-Ray-Modified Shocks. Peter Duffy. 90, 981, 16-A12 (1994)

EICHLER, DAvid. Particle Acceleration in High-Energy Gamma-Ray Sources. David Eichler. 90, 877, 14-F3 (1994)

Ekers, R. D. The Parkes-MIT-NRAO (PMN) Surveys. III. Source Catalog for the Tropical Survey $\left(-29^{\circ}<\delta<-9^{\circ} .5\right)$. Mark $R$. Griffith, Alan E. Wright, B. F. Burke, \& R. D. Ekers. 90, 179, 3-C6 (1994)

Ellison, Donald C. Monte Carlo Simulations of Particle Acceleration at Oblique Shocks. Matthew G. Baring, Donald C. Ellison, \& Frank C. Jones. 90, 547, 10-E6 (1994)

Eracleous, Michael. Double-peaked Emission Lines in Active Galactic Nuclei. Michael Eracleous \& Jules P. Halpern. 90, 1, 1-B1 (1994)

ERDós, G. Drift Acceleration at Interplanetary Shocks. G. Erdös \& A. Balogh. 90, 553, 10-F1 (1994)

FAHR, HANS-JORg. On the Asymmetry of the Distribution of the Anomalous Cosmic-Ray Component in the Heliosphere. Horst Fichtner, S. Ranga Sreenivasan, Hans-Jörg Fahr, Daniel Rucinski, \& Stanislaw Grzedzielski. 90, 595, 11-B9 (1994)

Fernandes, Francisco C. R. Microwave Type III-RS Bursts. Hanumant S. Sawant, Francisco C. R. Fernandes, \& José Angelo C. F. Neri. 90, 689, 12-C11 (1994)

Fernández-Figueroa, M. J. Ca II $\mathrm{H}$ and $\mathrm{K}$ and $\mathrm{H} \alpha$ Emissions in Chromospherically Active Binary Systems (RS Canum Venaticorum and BY Draconis). M. J. Fernández-Figueroa, D. Montes, E. De Castro, \& M. Cornide. 90, 433, 8-B6 (1994)

FICHTEL, CARL E. High-Energy Gamma-Ray Observations of Active Galaxies. Carl E. Fichtel. 90, 917, 15-B12 (1994)

FichtNer, HoRst. On the Asymmetry of the Distribution of the Anomalous Cosmic-Ray Component in the Heliosphere. Horst Fichtner, S. Ranga Sreenivasan, Hans-Jörg Fahr, Daniel Rucinski, \& Stanislaw Grzedzielski. 90, 595, 11-B9 (1994)

FIscher, PHILIPPE. A Search for Binaries in the Globular Cluster NGC 3201. Patrick Côté, Douglas L. Welch, Philippe Fischer, G. S. Da Costa, Peter Tamblyn, Patrick Seitzer, \& M. J. Irwin. 90, 83, 2-B5 (1994)

FRANK, ADAM. Oblique Magnetohydrodynamic Cosmic-Ray-modified Shocks: Two-Fluid Numerical Simulations. Adam Frank, T. W. Jones, \& Dongsu Ryu. 90, 975, 16-A4 (1994)

FRIDMAN, V. M. Efficiency for Electron Acceleration in Solar Energy Release Region as Estimated in the Context of Plasma Mechanism of Radio Emission. B. N. Levin, V. M. Fridman, \& O. A. Sheiner. 90 , 713, 12-E13 (1994)

Gallegos-Cruz, A. Weightiness of the Dispersive Rate in Stochastic Acceleration Processes. J. Pérez-Peraza \& A. Gallegos-Cruz. 90, 669, 12-B1 (1994)

Geroyannis, V. S. An Iterative Technique for Computing Rotating White Dwarf Models. V. S. Geroyannis \& P. J. Papasotiriou. 90 , 501, 9-A9 (1994)

Gopalswamy, N. Millimeter, Microwave, Hard X-Ray, and Soft X-Ray Observations of Energetic Electron Populations in Solar Flares. M. R. Kundu, S. M. White, N. Gopalswamy, \& J. Lim. 90, 599, 11-C1 (1994)

Solar Simple Bursts Observed with High Spectral Resolution in the 18-23 GHz Range. H. S. Sawant, R. R. Rosa, J. R. Cecatto, \& N. Gopalswamy. 90, 693, 12-D2 (1994)

Green, D. A. The Spectral Turnover of the "Filled-Center" Supernova Remnant 3C 58: Implications for When Acceleration Occurs. D. A. Green. 90, 817, 13-G12 (1994)

Gregory, P. C. The Parkes-MIT-NRAO (PMN) Map Catalog of Radio Sources Covering $-88^{\circ}<\delta<-37^{\circ}$ at $4.85 \mathrm{GHz}$. P. C.
Gregory, J. D. Vavasour, W. K. Scott, \& J. J. Condon. 90, 173, 3-B12 (1994)

Grenier, I. A. Gamma Rays from the Geminga Pulsar: Variations with Time and Phase. I. A. Grenier, K. Bennett, R. Buccheri, M. Gros, R. N. Henriksen, W. Hermsen, G. Kanbach, \& B. Sacco. 90 , 813, 13-G6 (1994)

Griffith, MARK R. The Parkes-MIT-NRAO (PMN) Surveys. III. Source Catalog for the Tropical Survey $\left(-29^{\circ}<\delta<-9^{\circ} .5\right)$. Mark R. Griffith, Alan E. Wright, B. F. Burke, \& R. D. Ekers. 90, 179, 3-C6 (1994)

Gros, M. Gamma Rays from the Geminga Pulsar: Variations with Time and Phase. I. A. Grenier, K. Bennett, R. Buccheri, M. Gros, R. N. Henriksen, W. Hermsen, G. Kanbach, \& B. Sacco. 90, 813, 13-G6 (1994)

GrZEDZIELSKI, STANISLAW. On the Asymmetry of the Distribution of the Anomalous Cosmic-Ray Component in the Heliosphere. Horst Fichtner, S. Ranga Sreenivasan, Hans-Jörg Fahr, Daniel Rucinski, \& Stanislaw Grzedzielski. 90, 595, 11-B9 (1994)

Godel, Manuel. Quiescent Microwave Emission from Late-Type Stars. Manuel Güdel. 90, 743, 13-A8 (1994)

HaERendel, Gerhard. Acceleration from Field-Aligned Potential Drops. Gerhard Haerendel. 90, 765, 13-C5 (1994)

HALPERN, JULES P. Double-peaked Emission Lines in Active Galactic Nuclei. Michael Eracleous \& Jules P. Halpern. 90, 1, 1-B1 (1994)

Hamilton, Russell J. Electrodynamics of Disk-accreting Magnetic Neutron Stars. M. Coleman Miller, Frederick K. Lamb, \& Russell J. Hamilton. 90, 833, 14-B4 (1994)

Disk-Accreting Magnetic Neutron Stars as High-Energy Particle Accelerators. Russell J. Hamilton, Frederick $K$. Lamb, \& M. Coleman Miller. 90, 837, 14-B9 (1994)

HamuY, M. The Tololo Nova Survey: Spectra of Recent Novae. $R$. E. Williams, M. M. Phillips, \& M. Hamuy. 90, 297, 5-F1 (1994)

HARDING, ALICE K. Gamma-Ray Burst Theory: Back to the Drawing Board. Alice K. Harding. 90, 863, 14-D13 (1994)

HaRtmann, Dieter H. Do Gamma-Ray Bursts Originate from an Extended Galactic Halo of High-Velocity Neutron Stars? Dieter $H$. Hartmann, Lawrence E. Brown, Lih-Sin The, Eric V. Linder, Vahe Petrosian, George R. Blumenthal, \& Kevin C. Hurley. 90, 893, 14-G10 (1994)

Henriksen, R. N. Gamma Rays from the Geminga Pulsar: Variations with Time and Phase. I. A. Grenier, K. Bennett, R. Buccheri, M. Gros, R. N. Henriksen, W. Hermsen, G. Kanbach, \& B. Sacco. 90, 813, 13-G6 (1994)

Hermsen, W. Gamma Rays from the Geminga Pulsar: Variations with Time and Phase. I. A. Grenier, K. Bennett, R. Buccheri, M. Gros, R. N. Henriksen, W. Hermsen, G. Kanbach, \& B. Sacco. 90, 813, 13-G6 (1994)

See Bennett, K., et al. Pulsar Studies with COMPTEL.

HildebrandT, J. Microwave Burst Timescales and Solar Flare Acceleration Processes. A. Krüger, B. Kliem, J. Hildebrandt, \& V. V. Zaitsev. 90, 683, 12-C3 (1994)

HiRAOKA, T. Gamma-Ray Spectral Observations with Yohkoh. M. Yoshimori, K. Suga, K. Morimoto, T. Hiraoka, J. Sato, K. Kawabata, \& K. Ohki. 90, 639, 11-F7 (1994)

Positron Annihilation Radiation from the 1991 November 15 Flare. K. Kawabata, M. Yoshimori, K. Suga, K. Morimoto, T. Hiraoka, J. Sato, \& K. Ohki. 90, 701, 12-D13 (1994)

Hofmann, A. The Flare of 1989 September 9 09:09 UT: Does Coronal Loop Collision Initiate Efficient Gamma-Ray Emission? H. Aurass, A. Hofmann, \& E. Rieger. 90, 707, 12-E6 (1994)

HURLEY, KEVIN. Observational Features of Cosmic Gamma-Ray Bursts: Evidence for Galactic versus Extragalactic Origin. Kevin Hurley. 90, 857, 14-D6 (1994)

HuRley, KEvin C. Do Gamma-Ray Bursts Originate from an Extended Galactic Halo of High-Velocity Neutron Stars? Dieter $H$. Hartmann, Lawrence E. Brown, Lih-Sin The, Eric V. Linder, Vahe Petrosian, George R. Blumenthal, \& Kevin C. Hurley. 90, 893, $14-\mathrm{G} 10$ (1994)

Hyung, S. Density Contrast Shell Models for the Planetary Nebula IC 2165. S. Hyung. 90, 119, 2-E4 (1994)

IKHSANOV, N. R. Particle Acceleration and Nonthermal Energy Release as an Effect of Magnetoactive Disk Accretion onto 
Gravitating Center. N. R. Ikhsanov \& L. A. Pustil'nik. 90, 959, 15-F12 (1994)

IRWIN, M. J. A Search for Binaries in the Globular Cluster NGC 3201. Patrick Cóté, Douglas L. Welch, Philippe Fischer, G. S. Da Costa, Peter Tamblyn, Patrick Seitzer, \& M. J. Irwin. 90, 83, 2-B5 (1994)

Janes, Kenneth A. Young Open Clusters as Probes of the Star Formation Process. I. An Atlas of Open Cluster Photometry. Randy L. Phelps \& Kenneth A. Janes. 90, 31, 1-D6 (1994)

Jones, Frank C. Monte Carlo Simulations of Particle Acceleration at Oblique Shocks. Matthew G. Baring, Donald C. Ellison, \& Frank C. Jones. 90, 547, 10-E6 (1994)

A Theoretical Review of Diffusive Shock Acceleration. Frank $C$. Jones. 90, 561, 10-F10 (1994)

JonES, T. W. Time-dependent Simulation of Cosmic-Ray Shocks, Including Alfvén Transport. T. $W$. Jones. 90, 969, 15-G10 (1994)

Oblique Magnetohydrodynamic Cosmic-Ray-modified Shocks: TwoFluid Numerical Simulations. Adam Frank, T. W. Jones, \& Dongsu Ryu. 90, 975, 16-A4 (1994)

Kafatos, Menas. Relativistic Particle Transport in Hot Accretion Disks. Peter A. Becker, Menas Kafatos, \& Michael Maisack. 90, 949, 15-E13 (1994)

KANBACH, G. Gamma Rays from the Geminga Pulsar: Variations with Time and Phase. I. A. Grenier, K. Bennett, R. Buccheri, M. Gros, R. N. Henriksen, W. Hermsen, G. Kanbach, \& B. Sacco. 90, 813, 13-G6 (1994)

KATZ-STONE, Debora M. Do Relativistic Electrons either Gain or Lose Energy, outside of Extragalactic Nuclei? Lawrence Rudnick, Debora M. Katz-Stone, \& Martha C. Anderson. 90, 955, 15-F6 (1994)

KaWABATA, K. Gamma-Ray Spectral Observations with Yohkoh. M. Yoshimori, K. Suga, K. Morimoto, T. Hiraoka, J. Sato, K. Kawabata, \& K. Ohki. 90, 639, 11-F7 (1994)

Positron Annihilation Radiation from the 1991 November 15 Flare. K. Kawabata, M. Yoshimori, K. Suga, K. Morimoto, T. Hiraoka, J. Sato, \& K. Ohki. 90, 701, 12-D13 (1994)

KIRK, J. G. Radio Emission from SNR 1987A. J. G. Kirk, P. Duffy, \& Lewis Ball. 90, 807, 13-F13 (1994)

KLIEM, B. Microwave Burst Timescales and Solar Flare Acceleration Processes. A. Krüger, B. Kliem, J. Hildebrandt, \& V.V.Zaitsev. 90, 683, 12-C3 (1994)

Kliem, Bernhard. Particle Orbits, Trapping, and Acceleration in a Filamentary Current Sheet Model. Bernhard Kliem. 90, 719, 12-F6 (1994)

KRUGER, A. Microwave Burst Timescales and Solar Flare Acceleration Processes. A. Krüger, B. Kliem, J. Hildebrandt, \& V. V. Zaitsev. 90 683, 12-C3 (1994)

KulJPERS, JaN. Strong Double Layers, Existence Criteria, and Annihilation: An Application to Solar Flares. Martin Volwerk \& Jan Kuijpers. 90, 589, 11-B2 (1994)

KuIPER, L. See BEnNETT, K., et al. Pulsar Studies with COMPTEL.

KundU, M. R. Millimeter, Microwave, Hard X-Ray, and Soft X-Ray Observations of Energetic Electron Populations in Solar Flares. M. R. Kundu, S. M. White, N. Gopalswamy, \& J. Lim. 90, 599, 11-C1 (1994)

Kundu, Mukul R. Pulsed Acceleration in Solar Flares. Markus J. Aschwanden, Arnold O. Benz, Brian R. Dennis, \& Mukul R. Kundu. 90, 631, 11-E11 (1994)

LAMB, FREDERICK K. Electrodynamics of Disk-accreting Magnetic Neutron Stars. M. Coleman Miller, Frederick K. Lamb, \& Russell J. Hamilton. 90, 833, 14-B4 (1994)

Disk-Accreting Magnetic Neutron Stars as High-Energy Particle Accelerators. Russell J. Hamilton, Frederick K. Lamb, \& M. Coleman Miller. 90, 837, 14-B9 (1994)

LANG, KENNETH R. Radio Evidence for Nonthermal Particle Acceleration on Stars of Late Spectral Type. Kenneth $R$. Lang. 90, 753, 13-B5 (1994)

LEVIN, B. N. Efficiency for Electron Acceleration in Solar Energy Release Region as Estimated in the Context of Plasma Mechanism of Radio Emission. B. N. Levin, V. M. Fridman, \& O. A. Sheiner. 90 , 713, 12-E13 (1994)

LICHTI, G. See BENNETT, K., et al. Pulsar Studies with COMPTEL.
LiM, J. Millimeter, Microwave, Hard X-Ray, and Soft X-Ray Observations of Energetic Electron Populations in Solar Flares. M. R. Kundu, S. M. White, N. Gopalswamy, \& J. Lim. 90, 599, 11-C1 (1994)

LINDER, ERIC V. Do Gamma-Ray Bursts Originate from an Extended Galactic Halo of High-Velocity Neutron Stars? Dieter $H$. Hartmann, Lawrence E. Brown, Lih-Sin The, Eric V. Linder, Vahe Petrosian, George R. Blumenthal, \& Kevin C. Hurley. 90, 893, 14-G10 (1994)

LOPEZ, RAMON E. Evidence for Particle Acceleration during Magnetospheric Substorms. Ramon E. Lopez \& Daniel N. Baker. 90, 531, 10-C13 (1994)

LUHR, H. Electron Acceleration at Quasi-parallel Shocks in the Solar Corona and Its Signature in Solar Type II Radio Bursts. G. Mann \& H. Lühr. 90, 577, 11-A1 (1994)

Macchetto, F. Hubble Space Telescope Observations of Synchrotron Jets. W. B. Sparks, J. A. Biretta, \& F. Macchetto. 90, 909, 15-B1 (1994)

MaISACK, MichaEl. Relativistic Particle Transport in Hot Accretion Disks. Peter A. Becker, Menas Kafatos, \& Michael Maisack. 90, 949 , 15-E13 (1994)

Mangalam, ARUn V. Effect of Advected Fields on Accretion Disk Dynamos. Arun V. Mangalam \& K. Subramanian. 90, 963, 15-G3 (1994)

MANN, G. Electron Acceleration at Quasi-parallel Shocks in the Solar Corona and Its Signature in Solar Type II Radio Bursts. G. Mann \& H. Lühr. 90, 577, 11-A1 (1994)

MazzITELLI, ITALO. New Pre-Main-Sequence Tracks for $M<2.5 M \odot$ as Tests of Opacities and Convection Model. Francesca $\bar{D}$ 'Antona \& Italo Mazzitelli. 90, 467, 8-D14 (1994)

MELroSE, D. B. Turbulent Acceleration in Solar Flares. D. B. Melrose. 90, 623, 11-E1 (1994)

Mentzel, M. Polarized One-Quantum Annihilation in Strong Magnetic Fields as a Process for Particle Deceleration in Neutron Star Atmospheres. D. Berg, M. Mentzel, \& G. Wunner. 90, 905, $15-A 10$ (1994)

MEYER, J. P. Energetic-Particle Abundances in Impulsive Solar Flare Events. D. V. Reames, J. P. Meyer, \& T. T. von Rosenvinge. 90, 649, 11-G7 (1994)

Miller, M. ColEman. Electrodynamics of Disk-accreting Magnetic Neutron Stars. M. Coleman Miller, Frederick K. Lamb, \& Russell J. Hamilton. 90, 833, 14-B4 (1994)

Disk-Accreting Magnetic Neutron Stars as High-Energy Particle Accelerators. Russell J. Hamilton, Frederick $K$. Lamb, \& M. Coleman Miller. 90, 837, 14-B9 (1994)

Mitrofanov, Igor G. Acceleration Processes for Gamma-Ray Bursts. Igor G. Mitrofanov. 90, 869, 14-E7 (1994)

Möbius, Eberhard. Sources and Acceleration of Energetic Particles in Planetary Magnetospheres. Eberhard Möbius. 90, 521, 10-C1 (1994)

MonTES, D. Ca $\| \mathrm{H}$ and $\mathrm{K}$ and $\mathrm{H} \alpha$ Emissions in Chromospherically Active Binary Systems (RS Canum Venaticorum and BY Draconis). M. J. Fernández-Figueroa, D. Montes, E. De Castro, \& M. Comide. 90, 433, 8-B6 (1994)

MORIMOTO, K. Gamma-Ray Spectral Observations with Yohkoh. M. Yoshimori, K. Suga, K. Morimoto, T. Hiraoka, J. Sato, K. Kawabata, \& K. Ohki. 90, 639, 11-F7 (1994)

Positron Annihilation Radiation from the 1991 November 15 Flare. K. Kawabata, M. Yoshimori, K. Suga, K. Morimoto, T. Hiraoka, J. Sato, \& K. Ohki. 90, 701, 12-D13 (1994)

Mueller, Cindy M. Cross Section Parameterizations for Cosmic-Ray Nuclei. II. Double Nucleon Removal. John W. Norbury \& Cindy M. Mueller. 90, 115, 2-D11 (1994)

Neri, José ÂNGELo C. F. Microwave Type III-RS Bursts. Hanumant S. Sawant, Francisco C. R. Fernandes, \& José Angelo C. F. Neri. 90, 689, 12-C11 (1994)

NorburY, JohN W. Cross Section Parameterizations for Cosmic-Ray Nuclei. II. Double Nucleon Removal. John W. Norbury \& Cindy M. Mueller. 90, 115, 2-D11 (1994) 
OHKI, K. Gamma-Ray Spectral Observations with Yohkoh. M. Yoshimori, K. Suga, K. Morimoto, T. Hiraoka, J. Sato, K. Kawabata, \& K. Ohki. 90, 639, 11-F7 (1994)

Positron Annihilation Radiation from the 1991 November 15 Flare. K. Kawabata, M. Yoshimori, K. Suga, K. Morimoto, T. Hiraoka, J. Sato, \& K. Ohki. 90, 701, 12-D13 (1994)

PAPASOTIRIOU, P. J. An Iterative Technique for Computing Rotating White Dwarf Models. V. S. Geroyannis \& P. J. Papasotiriou. 90, 501, 9-A9 (1994)

Pérez-Peraza, J. Weightiness of the Dispersive Rate in Stochastic Acceleration Processes. J. Pérez-Peraza \& A. Gallegos-Cruz. 90, 669, 12-B1 (1994)

Petrosian, VAHE. Do Gamma-Ray Bursts Originate from an Extended Galactic Halo of High-Velocity Neutron Stars? Dieter $H$. Hartmann, Lawrence E. Brown, Lih-Sin The, Eric V. Linder, Vahe Petrosian, George R. Blumenthal, \& Kevin C. Hurley. 90, 893, 14-G10 (1994)

Phelps, Randy L. Young Open Clusters as Probes of the Star Formation Process. I. An Atlas of Open Cluster Photometry. Randy L. Phelps \& Kenneth A. Janes. 90, 31, 1-D6 (1994)

PhILliPS, M. M. The Tololo Nova Survey: Spectra of Recent Novae. R. E. Williams, M. M. Phillips, \& M. Hamuy. 90, 297, 5-F1 (1994)

Protheroe, R. J. The Status of Ultra-High-Energy Gamma-Ray Observations. R. J. Protheroe. 90, 883, 14-F10 (1994)

PustiL'NIK, L. A. Particle Acceleration and Nonthermal Energy Release as an Effect of Magnetoactive Disk Accretion onto Gravitating Center. N. R. Ikhsanov \& L. A. Pustil'nik. 90, 959, 15-F12 (1994)

Reames, D. V. Energetic-Particle Abundances in Impulsive Solar Flare Events. D. V. Reames, J. R. Meyer, \& T. T. von Rosenvinge. 90 , 649, 11-G7 (1994)

ReYNolds, STEPHEN P. Model Images of Radio Halos around Supernova Remnants. Stephen P. Reynolds. 90, 845, 14-C6 (1994)

RIEGER, E. Gamma-Ray Precursors of Solar Flares. E. Rieger. 90, 645, 11-G1 (1994)

The Flare of 1989 September 9 09:09 UT: Does Coronal Loop Collision Initiate Efficient Gamma-Ray Emission? H. Aurass, A. Hofmann, \& E. Rieger. 90, 707, 12-E6 (1994)

RosA, R. R. Solar Simple Bursts Observed with High Spectral Resolution in the $18-23 \mathrm{GHz}$ Range. H. S. Sawant, R. R. Rosa, J. R. Cecatto, \& N. Gopalswamy. 90, 693, 12-D2 (1994)

RUCINSKI, DANIEL. On the Asymmetry of the Distribution of the Anomalous Cosmic-Ray Component in the Heliosphere. Horst Fichtner, S. Ranga Sreenivasan, Hans-Jörg Fahr, Daniel Rucinski, \& Stanislaw Grzedzielski. 90, 595, 11-B9 (1994)

RUDNICK, LAWRENCE. Do Relativistic Electrons either Gain or Lose Energy, outside of Extragalactic Nuclei? Lawrence Rudnick, Debora M. Katz-Stone, \& Martha C. Anderson. 90, 955, 15-F6 (1994)

Ryan, J. See BENNETT, K., et al. Pulsar Studies with COMPTEL.

RYU, DonGSU. Oblique Magnetohydrodynamic Cosmic-Ray-modified Shocks: Two-Fluid Numerical Simulations. Adam Frank, T. W. Jones, \& Dongsu Ryu. 90, 975, 16-A4 (1994)

SACCO, B. Gamma Rays from the Geminga Pulsar: Variations with Time and Phase. I. A. Grenier, K. Bennet, R. Buccheri, M. Gros, R. N. Henriksen, W. Hermsen, G. Kanbach, \& B. Sacco. 90, 813, 13-G6 (1994)

SATo, J. Gamma-Ray Spectral Observations with Yohkoh. M. Yoshimori, K. Suga, K. Morimoto, T. Hiraoka, J. Sato, K. Kawabata, \& K. Ohki. 90, 639, 11-F7 (1994)

Positron Annihilation Radiation from the 1991 November 15 Flare. K. Kawabata, M. Yoshimori, K. Suga, K. Morimoto, T. Hiraoka, J. Sato, \& K. Ohki. 90, 701, 12-D13 (1994)

SAWANT, H. S. Solar Simple Bursts Observed with High Spectral Resolution in the 18-23 GHz Range. H. S. Sawant, R. R. Rosa, J. R. Cecatto, \& N. Gopalswamy. 90,693, 12-D2 (1994)

Sawant, Hanumant S. Microwave Type III-RS Bursts. Hanumant $S$. Sawant Francisco C. R. Fernandes, \& José Angelo C. F. Neri. 90 , 689, 12-C11 (1994)

SCHLICKEISER, REINHARD. Cosmic-Ray Transport and Acceleration. Reinhard Schlickeiser. 90, 929, 15-D1 (1994)
On the Location of the Acceleration and Emission Sites in Gamma-Ray Blazars. Charles D. Dermer \& Reinhard Schlickeiser. 90, 945, 15-E7 (1994)

SchmitT, J. H. M. M. ROSAT Observations of Stellar Flares. J. H. M. M. Schmitt. 90, 735, 12-G12 (1994)

SCHÖNFELDER, V. See BENNETT, K., et al. Pulsar Studies with COMPTEL

ScotT, W. K. The Parkes-MIT-NRAO (PMN) Map Catalog of Radio Sources Covering $-88^{\circ}<\delta<-37^{\circ}$ at $4.85 \mathrm{GHz}$. P. C. Gregory, J. D. Vavasour, W. K. Scott, \& J. J. Condon. 90, 173, 3-B12 (1994)

SEITZER, PATRICK. A Search for Binaries in the Globular Cluster NGC 3201. Patrick Côté, Douglas L. Welch, Philippe Fischer, G. S. Da Costa, Peter Tamblyn, Patrick Seitzer, \& M. J. Irwin. 90, 83, 2-B5 (1994)

SERBER, A. V. Radiation-driven Acceleration in Photospheres of Nonaccreting Magnetic White Dwarfs. Vladimir V. Zheleznyakov \& A. V. Serber. 90, 783, 13-D13 (1994)

SHEINER, O. A. Efficiency for Electron Acceleration in Solar Energy Release Region as Estimated in the Context of Plasma Mechanism of Radio Emission. B. N. Levin, V. M. Fridman, \& O. A. Sheiner. 90, 713, 12-E13 (1994)

Sikora, MAREK. High-Energy Radiation from Active Galactic Nuclei. Marek Sikora. 90, 923, 15-C7 (1994)

SPARKS, W. B. Hubble Space Telescope Observations of Synchrotron Jets. W. B. Sparks, J. A. Biretta, \& F. Macchetto. 90, 909, 15-B1 (1994)

SREenivasan, S. Ranga. On the Asymmetry of the Distribution of the Anomalous Cosmic-Ray Component in the Heliosphere. Horst Fichtner, S. Ranga Sreenivasan, Hans-Jörg Fahr, Daniel Rucinski, \& Stanislaw Grzedzielski. 90, 595, 11-B9 (1994)

Strong, A. See Bennett, K., et al. Pulsar Studies with COMPTEL

Subramanian, K. Effect of Advected Fields on Accretion Disk Dynamos. Arun V. Mangalam \& K. Subramanian. 90, 963, 15-G3 (1994)

SUGA, K. Gamma-Ray Spectral Observations with Yohkoh. M. Yoshimori, K. Suga, K. Morimoto, T. Hiraoka, J. Sato, K. Kawabata, \& K. Ohki. 90, 639, 11-F7 (1994)

Positron Annihilation Radiation from the 1991 November 15 Flare. K. Kawabata, M. Yoshimori, K. Suga, K. Morimoto, T. Hiraoka, J. Sato, \& K. Ohki. 90, 701, 12-D13 (1994)

SYNIAVSKII, D. V. Electron-Beam Evolution in Partially Ionized Hydrogenic Plasma with Return Currents. D. V. Syniavskii \& $V . V$. Zharkova. 90, 729, 12-G4 (1994)

TAMblyn, Peter. A Search for Binaries in the Globular Cluster NGC 3201. Patrick Côté, Douglas L. Welch, Philippe Fischer, G. S. Da Costa, Peter Tamblyn, Patrick Seitzer, \& M. J. Inwin. 90, 83, 2-B5 (1994)

TaVAnI, Marco. Relativistic Particle Acceleration in Plerions. Jonathan Arons \& Marco Tavani. 90, 797, 13-F1 (1994)

THE, LiH-Sin. Do Gamma-Ray Bursts Originate from an Extended Galactic Halo of High-Velocity Neutron Stars? Dieter $H$. Hartmann, Lawrence E. Brown, Lih-Sin The, Eric V. Linder, Vahe Petrosian, George R. Blumenthal, \& Kevin C. Hurley. 90, 893, 14-G10 (1994)

Tokunaga, Alan T. Stellar Density Enhancements Associated with IRAS Sources in L1641. Hua Chen \& Alan T. Tokunaga. 90, 149, 2-G12 (1994)

UlMER, M. P. Gamma-Ray Observations of Pulsars. M. P. Ulmer. 90, 789, 13-E6 (1994)

VANDAS, M. Acceleration of Electrons at a Curved Shock. M. Vandas. 90, 583, 11-A8 (1994)

VAVAsouR, J. D. The Parkes-MIT-NRAO (PMN) Map Catalog of Radio Sources Covering $-88^{\circ}<\delta<-37^{\circ}$ at $4.85 \mathrm{GHz}$. $P$. $C$. Gregory, J. D. Vavasour, W. K. Scott, \& J. J. Condon. 90, 173, 3-B12 (1994)

VILMER, N. Solar Hard X-Ray and Gamma-Ray Observations from Granat. N. Vilmer. 90, 611, 11-D1 (1994)

VolWERK, MARTIN. Strong Double Layers, Existence Criteria, and Annihilation: An Application to Solar Flares. Martin Volwerk \& Jan Kuijpers. 90, 589, 11-B2 (1994) 
VOn RosenVInge, T. T. Energetic-Particle Abundances in Impulsive Solar Flare Events. D. V. Reames, J. P. Meyer, \& T. T. von Rosenvinge. 90, 649, 11-G7 (1994)

Welch, Douglas L. A Search for Binaries in the Globular Cluster NGC 3201. Patrick Cote, Douglas L. Welch, Philippe Fischer, G. S. Da Costa, Peter Tamblyn, Patrick Seitzer, \& M. J. Inwin. 90, 83, 2-B5 (1994)

WHITE, S. M. Millimeter, Microwave, Hard X-Ray, and Soft X-Ray Observations of Energetic Electron Populations in Solar Flares. M. R. Kundu, S. M. White, N. Gopalswamy, \& J. Lim. 90, 599, 11-C1 (1994)

Williams, R. E. The Tololo Nova Survey: Spectra of Recent Novae. R. E. Williams, M. M. Phillips, \& M. Hamuy. 90, 297, 5-F1 (1994)

Wright, Alan E. The Parkes-MIT-NRAO (PMN) Surveys. III. Source Catalog for the Tropical Survey $\left(-29^{\circ}<\delta<-9^{\circ} .5\right)$. Mark R. Griffith, Alan E. Wright, B. F. Burke, \& R. D. Ekers. 90, 179, 3-C6 (1994)

Wunner, G. Polarized One-Quantum Annihilation in Strong Mag- netic Fields as a Process for Particle Deceleration in Neutron Star Atmospheres. D. Berg, M. Mentzel, \& G. Wunner. 90, 905, 15-A10 (1994)

YoSHIMORI, M. Gamma-Ray Spectral Observations with Yohkoh. M. Yoshimori, K. Suga, K. Morimoto, T. Hiraoka, J. Sato, K. Kawabata, \& K. Ohki. 90, 639, 11-F7 (1994)

Positron Annihilation Radiation from the 1991 November 15 Flare. K. Kawabata, M. Yoshimori, K. Suga, K. Morimoto, T. Hiraoka, J. Sato, \& K. Ohki. 90, 701, 12-D13 (1994)

ZAITSEV, V. V. Microwave Burst Timescales and Solar Flare Acceleration Processes. A. Krüger, B. Kliem, J. Hildebrandt, \& V. V. Zaitsev. 90, 683, 12-C3 (1994)

ZHARKoVA, V. V. Electron-Beam Evolution in Partially Ionized Hydrogenic Plasma with Return Currents. D. V. Syniavskii \& V. V. Zharkova. 90, 729, 12-G4 (1994)

ZhelezNyakov, VladimiR V. Radiation-driven Acceleration in Photospheres of Nonaccreting Magnetic White Dwarfs. Vladimir $V$. Zheleznyakov \& A. V. Serber. 90, 783, 13-D13 (1994) 